\title{
Poésie et Spiritualité
}

\author{
Jean-Pierre Siméon \\ Institut Universitaire, Clermont-Ferrand
}

Je n'hésiterai pas à engager mon propos, a risque de le disqualifier d'emblée, sur cette citation de Pierre-Jean Jouve : "Nous ne savons pas ce que c'est que la poésie et tout poème, s'il est vrai, demeure mystère." Ni boutade ni prudence, cette manière de faire veut seulement signaler que s'agissant de la poésie, nous avançons toujours en terre incertaine, tout pas risquant le démenti de celui qui suivra, dès lors qu'on ne parle pas c'est ce que j'entends faire, à partir de l'autorité d'un savoir mais du point de vue de l'expérience. Je ne vous entretiens pas ici d'un genre littéraire de toute façon problématique, mais d'une chose dont l'étendue et la substance sont variables à l'extrême, qui a voir comme l'indique la formule de Jouve avec la vérité et le mystère et qui, en ce qui me concerne, représente au même titre que l'expérience amoureuse j'en dis déjà long, n'est-ce pas, en marquant cette équivalence le monde privilégié de ma relation au monde. Il se pourrait au reste que, revendiquant scandaleusement de ne pas définir la chose dont je vais parler, je légitime paradoxalement le thème de mon discours : poésie et spiritualité. Je veux dire que si nous perdons toujours à vouloir définir la poésie en elle-même, nous aurons par contre quelque chance d'y parvenir en faisant apparaître ses contours, c'est-à-dire en inventoriant ce qui lui est contigu d'une part, ce qui la nie d'autre part. Aussi poésie spiritualité, poésie et langage, poésie et engagement, poésie et magie, poésie et rationalité, poésie et... mais on voit bien sûr que la série est à l'infini et que, hormis quelques rares incongruités absolues qui tombent sous le sens comme poésie et mécanique automobile ou poésie et contrôle de naissances, rien ou presque de ce qui touche au geste, à la pensée et à l'environnement de l'homme ne lui est étranger. J'aurais ainsi pu parler, sans démériter peut-être, de poésie et vie quotidienne, poésie et capitalisme, voire poésie et rêve de vacances si l'on veut bien ici admettre avec Jean Tardieu, récemment parti chez la Môme Néant, que la poésie a aussi à voir avec l'attrait du vide. 
En fait nous avançons. Car si je dis que tout a à voir avec la poésie, j'entends réciproquement que la poésie a à voir avec tout, je dirais mieux avec le tout du monde. Voilà sa vérité et son mystère. Pour que ce soit possible, il faut bien qu'elle manifeste, en s'exprimant, quelque dénominateur commun, que proprement elle dénomme un lieu commun, à tout ce qui est, un enjeu unanime, quelque unité improbable, en effet, autrement dispersé dans l'afflux exubérant du réel. De là peut-être l'universalité et l'intemporalité de la poésie dans l'histoire des hommes. Elle est la dernière chose dont une société humaine se passerait. On se souvient du chapitre bouleversant de Si c'est un homme où Primo Levi, au coeur de l'abjection d'Auschwitz, se remémore désespérément les chants de Dante. Essentielle et majeure incantation : elle témoigne de ce voeu éperdu d'être, d'être encore et simplement un homme contre ce qui justement nie 1'homme dans son essence même. Certains déportés, dit Levi, payaient de ce qu'ils avaient de plus précieux, une ration de pain, celui qui chantait pour eux, attitude qui est à mes yeux l'équivalent strict dans un autre registre culturel de l'évocation du Dante par Levi. Je vois décidément pour ma part dans ce recours au poème et au chant dans une expérience limite la manifestation exemplaire que la poésie est une des plus ultimes, des plus irréductibles preuves de notre présence au monde, de cette pure vertu d'exister que tout menace et que le poète n'a de cesse d'évoquer par le pouvoir proprement magique de sa parole.

Incantation, évocation, magie : je n'ai bien sûr pas employé par hasard ces mots dont je sais le poids. Loin de moi la tentation de réduire la poésie à un irrationnel de pacotille ou à quelque spiritisme cultivé qui, via la voyance et les transes de la Pythie, la renverrait au prophétisme romantique qui est comme la caricature de ce dont il s'agit en fait. Le poème en outre n'est pas un grigri qu'on agite face à l'épouvante des temps.

De quoi s'agit-il donc? Du rapport singulier que l'homme entretient avec le réel par le langage. Au fil de son évolution, de ses avatars culturels et géographiques, la parole n'a rien perdu de son affinité naturelle avec le carmen primitif, ce charme par quoi l'homme, dépourvu devant absence perpétuellement renouvelée du réel cherche à instituer un pouvoir sur le monde.

Mode d'appropriation et de métamorphose, la parole poétique nomme et représente ce qui n'est plus ou n'est pas encore pour le posséder et le préserver. Une chose n'existe que si elle est nommée et c'est en quoi la poésie née de la sollicitation du réel lui confère en retour sa validité et peut-être la grâce d'une éternité. Et c'est en 
quoi le poète nommant par le signe, le nom, la métaphore, le symbole et le mythe, l'insaisissable qu'il habite assume une attitude proprement magique et fait apparaître à la conscience ce qui lui échappe. On peut comprendre ainsi la formule d'André Breton : "L'imaginaire est ce qui tend à devenir réel." Les mots du poète fondent un monde, comme le font les mots de l'enfant, comme le fit dans l'éclair des premières fois l'humanité naissante.

Tâche inlassable cependant, toujours insuffisante, toujours déçue peut-être puisque le réel, n'est-ce pas, s'augmente à chaque instant de ce qu'il n'est pas et à chaque instant se perd. "Nous avons perpétuellement à nous donner le monde où nous sommes," dit magnifiquement le philosophe Stanislas Breton. Chaque poème recommence ce don et l'on imagine à quelle perte de soi, et à quel terrible abandon du réel s'exposent les sociétés qui se détournent de leurs poètes. Le pire les guette et notamment cette perversion généralisée du langage à quoi nous assistons quotidiennement dans le monde occidental. Alors que la parole du poète, convoquant les caractères les plus mystérieux de la réalité, l'interrogeant sans relâche et sans relâche inventant des moyens adéquats à sa complexité, tente éperdument de combler le vide qui sépare le mot de la chose, les langues de bois à présent dominantes creusent ce vide et de cet écart vertigineux fondent leur pouvoir. Système de représentation autotélique et artificiel, tragiquement coupé de l'expérience, ces langues qui ne parlent plus car la parole est justement la fleur d'une expérience accomplissent une oeuvre terrifiante de déréalisation et le miroir d'Alice naguère traversé par les aventuriers du rêve n'est plus qu'un miroir aux alouettes qui renvoie plus à l'homme que le reflet de ses mirages.

Croyez-vous que vous entretenant de cette perdition, j'oublie ce qui doit faire l'objet de mon propos, à savoir l'analyse de ce que se doivent mutuellement poésie et spiritualité ? Pas un instant. Parce que ce qui se perd dans la parole perdue ce sont définitivement les privilèges du coeur et de l'esprit, ce sentiment et cette intelligence du monde qui s'instruisent du mystère même qu'ils tentent passionnément de nommer. Ainsi disait Mallarmé : "La poésie est l'expression par le langage humain ramené à son rythme essentiel du sens mystérieux des aspects de l'existence : elle doue ainsi d'authenticité notre séjour et constitue la seule tâche spirituelle."

Qu'est-ce donc ce mystère auquel Jouve d'abord, Mallarmé maintenant, renvoient la poésie? Chaque poète a sa réponse. Pour moi, cela ne fait nul doute : c'est ce trou noir, ce point d'achoppement qui de Baudelaire à Rimbaud, de Breton à Char, de Reverdy à Bonnefoy, d'Eluard à Saint John Perse, tire la poésie vers un au-delà du réel 
sans jamais pourtant, puisque pour ce siècle Dieu est mort, l'entraîner dans l'absolu d'une transcendance. Il s'agirait d'un invisible coexistant au visible et que celui-ci inclut sans le nommer ou du moins si indirectement qu'il faut à sa perception l'effort d'une attention extrême. Il s'agirait d'un indéfini dans le fini. Quelque chose dans la nature du profane mais qui dans son mode d'apparition, dévoilement ou révélation, en appelle au sacré. Un au-delà du réel au coeur même du réel, mais consubstantiel au réel, de lui tributaire et l'informant en retour de sa résonance énigmatique. Un "horla" qui ne saurait se réduire au maléfice imaginé par Maupassant, le Gouffre pour Baudelaire, le là-bas de Rimbaud, le surréel de Breton, l'énigme pour Char, la présence pour Bonnefoy, pour Bobin la merveille et l'obscur, pour Chedid ce plus loin dont on ne sait le nom, et pour Eluard sans doute l'amour.

Toutes façons de nommer l'innommable, un dépassement possible, parce que pressenti, de l'évidence et de la réalité immédiate qui autorisait Saint John Perse à affirmer qu'en ce siècle la poésie avait remplacé la métaphysique. On ne peut manquer ici de rappeler la parole de Novalis : "La poésie est la religion originelle de l'humanité," parole qui inverse la distribution traditionnelle des termes donnant pour modèle à la religion ce qui lui préexiste et ne lui appartient pas. Voilà qui fonde à mes yeux une spiritualité je dirai profane et laïque qui ouvre donc la réalité à autre chose qu'elle même, un ailleurs dans l'ici-bas que les poètes pressentent comme un mystère quotidien, habitable, mais qu'ils ne prétendent jamais au rebours de la philosophie ou de la théologie, expliquer ni même comprendre. Ou bien comprendre ici serait à entendre dans le sens étymologique d'embrassement : les mots du poème révèlent selon l'expression de Roberto Jurroz "une réalité totale" extensive, ce que pour ma part j'ai nommé, si vous me permettez de me citer "l'illimité dans l'homme." Comprendre le réel, c'est-à-dire étreindre en vue d'une saisie globalisant l'objet et son double de mystère, la matière et son être perçus non pas de façon contradictoire selon le monde platonicien mais dans une inclusion mutuelle, telle est à mes yeux la tâche assignée au poète. On voit bien que c'est manière de reconsidérer le réel hors des canons antagonistes du spiritualisme et du matérialisme. N'oublions pas que la plupart des poètes que j'ai cités en référence sont athées ou agnostiques et $\mathrm{j}$ 'ai par exemple toujours pensé que c'était l'introuvable réconciliation de ces contraires, la matière et la conscience, la chair et l'esprit, qu'avait tenté le surréalisme. Il revient à Nietzsche, on le sait, d'avoir basculé la vieille hiérarchie du dualisme qui assure la primauté de l'âme sur le corps, de la pensée sur la sensation, de la raison sur les passions, du divin sur l'humain. Il revient je crois aux poètes de ce temps de tenir dans un rapport d'égalité réciproque ce que l'idéalisme et le sensualisme distinguaient dans une 
mutuelle exclusion. Si René Char et beaucoup d'autres cherchent avec Heidegger "la fondation de l'être par la parole," on ne peut ignorer que leur parole est plus qu'en tout autre temps incarnée, elle est une voix d'homme qui s'élève à partir des contingences concrètes, nourrie de la merveille et de la tragédie éprouvées dans un engagement qui est le ca-ractère le plus constant des poètes de ce siècle. Il ne s'agit pas ici, ou pas seulement, de l'engagement social et politique de la personne, mais de cet enracinement de la parole poétique dans l'expérience, au sens où Rilke le manifeste, je dis bien : dans la chair de l'expérience. Ecoutons Rilke :

Les vers ne sont pas, comme certains croient, des sentiments (on les a toujours assez tôt), ce sont des expériences. Pour écrire un seul vers, il faut avoir vu beaucoup de villes, d'hommes et de choses, il faut connaître les animaux, il faut sentir comment volent les oiseaux et savoir quels mouvements font les petites fleurs en s'ouvrant le matin. Il faut pouvoir repenser à des chemins dans des régions inconnues, à des rencontres inattendues, $\grave{a}$ des départs que l'on voyait longtemps approcher, ̀̀ des jours d'enfance dont le mystère ne s'est pas encore éclairci, à ses parents qu'il fallait qu'on froissât lorsqu'ils vous apportaient une joie et qu'on ne la comprenait pas (c'était une joie faite pour un autre), à des maladies d'enfance qui commençaient si singulièrement par tant de profondes et graves transformations, à des jours passés dans des chambres clames et contenues, à des matins au bord de la mer, à la mer elle-même, à des mers, à des nuits de voyage qui frémissaient très haut et volaient avec toutes les étoiles, et il ne suffit même pas de savoir penser à tout cela. Il faut avoir le souvenir de beaucoup de nuits d'amour, dont aucune ne ressemblait à l'autre, de cris de femmes hurlant en mal d'enfant, et de légères, de blanches, de dormantes accouchées qui se refermaient. Il faut encore avoir été auprès de mourants, être resté assis auprès de morts, dans la chambre, avec la fenêtre ouverte et les bruits qui venaient par a-coups. Et il ne suffit même pas d'avoir des souvenirs. Il faut savoir les oublier quand ils sont nombreux, et il faut avoir la grande patience d'attendre qu'ils reviennent. Car les souvenirs eux-mêmes ne sont pas encore cela. Ce $n$ 'est que lorsqu'ils deviennent en nous sang, regard, geste, lorsqu'ils $n$ 'ont plus de nom et ne se distinguent plus de nous, ce n'est qu'alors qu'il peut arriver qu'en une heure très rare, du milieu d'eux, se lève le premier mot d'un vers. (Les cahiers de M. L. Bridge) 
Le poète dès lors, avant d'être homme de langage est d'abord un homme tout simplement et l'authenticité de la parole se légitime de l'authenticité de son expérience humaine. Ce que dit ainsi Andrée Chedid, dont le lyrisme généreux est aujourd'hui un de nos biens les plus précieux: "Les habiles, les jongleurs de mots sont plus éloignés de la poésie que cet homme qui sans parole aucune se défait de sa journée, le regard levé vers un arbre, ou le coeur attentif à la voie d'une âme." Oui, le poète est un homme engagé, c'est-à-dire scrupuleusement et passionnément attentif à ces riens de l'existence en quoi il sait lire l'essentielle part d'énigme qu'ils recèlent, en quoi il sait reconnaitre "la fleur originelle" parce qu'il sait que toute chose manifeste, dans le frémissement de l'être, le sens premier de la vie.

J'ai dit "sens:" je tire, voyez-vous, le fils les uns après les autres. Pour quoi d'autre le poète s'abandonnerait-il à la pratique du mystère si ce n'était pour faire apparaître ce sens dont on nous dit aujourd'hui à l'envi que nous serions, définitivement peutêtre, dépourvus. Ici je commencerai par dire, parodiant la formule de Jouve, que je ne sais pas ce que c'est que le sens et que tout sens s'il est vrai demeure mystère. Ce qui importe après tout c'est d'être saisi, dans l'action du poème, de la conviction qu'un sens est là, dans le tout des choses où nous vivons, et qu'il est approchable sinon saisissable. C'est une autre manière d'invoquer, vous voudrez bien passer sur la maladresse de l'expression, cette transcendance interne dont $\mathrm{j}$ 'essayais de dire quelque chose tout à l'heure. Tout poème, s'il est vrai, nous donne la prémonition bouleversante d'un inconnu, ce que Char nomme "l'inconnu devant soi." Il y a fort à parier que ce bouleversement soit la cause et la fin de toute poésie. Une émotion, un arrachement à l'insignifiance apparente du monde qui, dans l'instant, nous projettent, stupéfaits, dans le lieu d'un sens possible, une utopie. Quant au sens, le poète jamais ne donne de réponses, mais veilleur infatigable, continuant obstinément d'interroger lorsque tous autour de lui ont abdiqué (tous ou presque: il se trouve des alliés dont manifestement vous êtes), il nous préserve de l'absurde. A tous ceux, les dépossédés, qui errent aujourd'hui parmi les décombres du sens total, unique et obligatoire, le poète offre sinon la clef du moins un chemin: "Comment vivre sans inconnu devant soi?" demande René Char et c'est un programme de vie.

On me dit parfois que les hiéroglyphes du poète n'ont rien à dire aux hommes et aux femmes de ce temps, que leur parole, retranchée dans une abstraction égoïste, ignorerait le souci quotidien du commun des mortels. C'est de mon point de vue un douloureux malentendu. Il est la conséquence du préjugé courant, naïf mais tenace, qui veut que, hors de la compréhension religieuse du monde, la réalité se éréduise à 
l'évidence objective et que le seul discours sur l'événement, le fait, la chose obvie en rendrait compte. C'est par préjugé rationaliste refuser à la réalité ce double fond mystérieux qui requiert spécifiquement la poésie. Qui ne voit que si les mots chômage, SIDA, kalachnikov, etc., ne se trouvent que rarement dans les poèmes, l'essentielle douleur dont ils ne sont que les symptômes partiels et transitoires est toute la préoccupation des poètes? Rappelons-nous encore ce que disait Primo Levi dont l'oeuvre est enseignement crucial et irréductible: quelle que soit l'atrocité des conditions d'une existence, ce n'est que dans la prémonition d'un éventuel dépassement de la réalité où il vit qu'un homme peut préserver sa qualité d'homme. A-t-on bien entendu la capitale leçon qu'il nous donne quand il dit devoir sa survie à la survie en lui de cet "éventuel", braise ultime là où tout meurt. Plus pré-gnantes sont la pesée de l'absurde et la conviction de l'impasse, plus urgente, plus nécessaire est la position de qui s'entête à préserver la certitude d'un autre possible par-delà les apparences. Ecrire un poème aujourd'hui, c'est-à-dire donner la chance d'apparition à des sens virtuels, c'est-à-dire désigner et recueillir la moindre clarté dans l'opacité des ombres, c'est résister au désaveu que l'absurde inflige à l'homme, c'est manifester la plus incontestable des compassions envers ses frères comme la plus intransigeante des révoltes. Tout poète, même le poète du dimanche, usant du langage pour appeler les faits de son existence à cet autrement qu'il pressent en eux c'est la raison même de son geste d'écrire témoigne d'un refus majeur, le refus d'en rester aux limites du connu et du tangible, refus qui est dans le même temps l'affirmation d'une liberté souveraine, qu'il dessine la courbe d'une colline dans les tiédeurs du soir, qu'il évoque l'éclat tendre d'un baiser, la rumeur de ses pas dans le silence ou le déni que le rêve oppose à sa nuit, tout poète nous dit que le monde n'est pas que le monde et refonde pour l'heure qui vient, notre ardeur à vivre. Il est "éveilleur de sens", mais d'un sens multiple, foisonnant, contradictoire, qui ne se soumet à nul à priori idéologique, esthétique ou moral puisque prenant son origine dans la chair et le sang de l'expérience, dans l'épreuve quotidienne, humaine, terriblement humaine du désir et de l'effroi. Pensons ici à l'oeuvre radicale d'Antonin Artaud, et à ce que son cri signifie d'exigence, appel insensé du sens d'un homme que rien ne console de sa privation. Ce qui donne sens à ma vie, je le dis sans forfanterie, c'est, face à la certitude de la mort et au coeur des édésarrois, la poésie et l'amour, parce que tous deux opèrent en moi ce "ravissement" où dans l'émoi de l'instant se reconnaît la clarté mystérieuse d'un sens dont il m'importe peu de ne rien savoir d'autre qu'il existe.

Je prévois bien sûr l'objection sinon les ricanements. Voilà dira-t-on, comme une mystique qui ne dit pas son nom. Je conviens qu'il y a quelque affinité d'apparence. 
"Pour moi, dit Roberto Juarroz, la mystique est l'expérience de l'occulte, de la nonévidence, du tout par la voie de l'intériorité, le sentiment de faire partie du tout. Une expérience très proche, me semble-t-il de la poésie." Affinité il est vrai, proximité peut-être, identité sûrement pas. Car par le truchement de l'ascèse, le mystique s'arrache au réel pour atteindre à l'ordre divin où il se perd. Au rebours l'expérience poétique, comme l'expérience amoureuse, ne nous ôte à la contingence que pour nous porter au plein coeur du réel, dans sa plénitude parfaite et réconciliée. Loin d'être une ascèse, elles sont une immersion par quoi nous touchons à quelque absolu du réel où se comprennent et se résolvent les contraires, le corps et l'esprit, le visible et l'invisible, l'autre et le même. S'il est vrai qu'à travers les ghazels la mystique arabe la plus ancienne emprunte les voies de la poésie et de l'amour, je n'en conclus pour ma part qu'à une équivalence dans les modalités d'un dépassement qui ne vise par ailleurs pas tout à fait la même énigme, qui ne la situe pas en tout cas au même lieu dans la hiérarchie des songes. C'est la raison pour laquelle Louis Aragon dont on n'ignore pas la préférence pour le matérialisme marxiste dont je me sens pour ma part également très proche, a pu dans Le fou d'Elsa emprunter les modes de la mystique. Ce n'est bien sûr par hasard s'il s'agit dans ce livre d'un chant d'amour extrême. Je dirais à la limite qu'on ne peut concevoir de poésie amoureuse, c'est-à-dire comme une étreinte passionnée $d$ monde, et d'amour que poétique, c'est-à-dire créateur de l'être. En effet cette quête d'un sens mystérieux par les voies d'un dépassement de la réalité immédiate fonde une spiritualité au sens le plus exigeant du terme. Mais il faut admettre contre nos habitudes de penser qu'elle peut faire l'économie de la divinité comme de la religiosité et surtout qu'elle puisse paradoxalement récuser l'antique antagonisme du corps et de l'esprit. J'irais jusqu'à dire pour renverser les choses que ce mode d'accès par le langage ramené à son rythme essentiel au sens mystérieux du monde est essentiellement poétique et que ce n'est que de seconde main qu'un Saint Jean de la Croix peut se l'approprier comme un moyen efficace de sa quête religieuse. Voilà pourquoi bien des athées peuvent le lire comme un poète, comme ils peuvent lire, sans l'idée de Dieu, le Cantique des Cantiques: c'est leur expérience humaine qui s'en trouve augmentée et leur compréhension du réel qui s'en trouve enrichie ici et maintenant. Nullement réductrice, en dépit de leur mécréance, leur lecture est parfaitement égale dans la quantité de sens qu'elle récupère à celle du croyant.

"L'amour la poésie" donc. Ou encore: "Poésie la vie entière." Voici deux formules empruntées à René Guy Cadou qui signifient clairement à mes yeux les voies et les enjeux de la saisie poétique. Bien sûr l'amour dont je parle ici, inclut mais excède le geste amoureux ordinaire et $\mathrm{j}$ 'y vois quant à moi un mode fondamental de 
relation au monde, qui en appelle donc dans sa faim vertigineuse de tendresse et de consolation à l'infini des êtres et des choses.

Sans doute est-il bon que le discoureur laisse ici la place au poète. Permettez-moi de vous lire cet extrait du cinquième chant du Sentiment du monde qui est éloge amoureux autant qu'éloge de l'amour:

L'amour, peut-être, n'est pas ce dont je parle, c'est d'un plus grand vouloir que je parle, brassant océans et montagnes, chaumes et moissons

L'imprécation d'un songe, les nuits parentes de la terre et de l'oubli, espaces de la tendresse, Le corps à corps des arbres et des soleils, Et l'art terrible de l'immense Cela c'est le désir de l'amour, Son pas posé à l'aventure, unissant l'astre et l'abîme, Accordant la beauté aux pauvres d'entre les pauvres, Cela c'est l'âme de l'amour, Comme l'abeille incessante et gourmande est l'âme de la fleur et la raison de

sa couleur

Je parle d'un amour plus vaste que l'amour, D'un fleuve qui embrasse l'invisible du fleuve, l'éblouissement sur l'eau verte,

ô forme imprévue de l'impatience!

Je voudrais qu'on entende ces vers comme le rapport d'une expérience simple, banale, dont chacun d'entre vous, j'en suis sûr a la partage puisque si vous êtes là ce n'est que par une disponibilité à comprendre, donc à aimer. Quelle est elle cette expérience? Celle du mouvement qui porte vers le monde, d'une volonté d'accueil qui subsume la dispersion des jours et la multitude des circonstances. Cette "forme imprévue de l'impatience," c'est cela l'amour, la poésie, et cela ne requiert d'autre aptitude q'une attention imprévoyante et confiante à l'autre. 
La disposition intérieure à saisir poétiquement et amoureusement le monde, c'est cela que pour ma part je nomme l'âme. Disposition intellectuelle et morale, soit, mais pour moi, avant tout sensuelle, devant tout à une érotisation de la perception parce que compromise avec la chair désirante, matière inclinée vers le sens. La pensée matérialiste n'a pas tort de se débarasser d'une âme qui sent trop le mépris du terrestre et le dépit des contraintes qu'il assume, mais elle aurait tort tout autant de ne pas donner asile à ce qui, hors de la raison discursive, , agit en l'homme et fonde son élan non pas seulement vers la vie ce n'est que l'instinct de conservation mais vers la louange, ce qui ne sert à rien qu'à être, si l'on peut dire, davantage. L'âme donc serait, enracinée dans le trivial de l'expérience, cette émotion poétique dont parle Reverdy, Orient fulgurant dont procède notre effort vers le monde. Son destin n'est pas l'élévation mais la traversée et la poésie qui la manifeste sera horizontale, à hauteur d'homme, comme peut-être, par métaphore, les vers que j'ai lus du Sentiment $d u$ monde le proposent. C'est cette position que je reconnais dans le propos d'Andrée Chedid que je ne cite si volontiers que parce que à bien des égards mes pas suivent les siens: "Le poème se nourrit de mouvements; mouvements de cet être intérieur que certains appelleraient "âme." Son rythme est celui de la vague, son dessein est de traverser."

Il faudrait, au fond, pour illustrer le syncrétisme que je tente dans ces réflexions, inventer au prix d'un oxymore monstrueux et qui enfreint, cela va de soi, le philosophiquement correct "un matérialisme spirituel" puisque nous cherchons à tenir ce paradoxe que la poésie est un enracinement dans l'être par un cheminement dans la matière du réel. J'ai quelques scrupules à me référer au philosophe et théologien Stanislas Breton pour donner une pertinence à ce qui n'est somme toute qu'une lubie de poète, mais le double concept de l'Etre-dans et de l'Etre-vers qu'il expose dans Poétique du sensible me convient trop parfaitement. Je ne saurais bien évidemment lui faire endosser les partis pris qui sont les miens, la rigueur et l'exigence de sa pensée ne le supporteraient pas. Stanislas Breton suppose donc l'homme confronté à deux modalités de l'être, l'Etre-dans et l'Etre-vers, dans une alternance aussi nécessaire que contradictoire. La demeure et le voyage en pourraient être les figures primordiales. Celle-là appelle 1'enracinement, la fixité, la stabilité, celui-ci le parcours, le devenir et la mutation. Si l'une s'assume sur le mode intransitif "un agir qui ne fait rien, un agir profond qui consiste à se tenir là", l'autre sur le mode transitif est élan et passage. Inscrit dans l'ouverture et un sentir qui s'ouvre sur ce qui le change, l'Etre-vers est caractérisé par l'exotropisme, tentation de l'ailleurs et de l'autre. Puisqu'il ne s'agit pas d'entendre ces deux modalités comme exclusives l'une de l'autre mais de les envisager dans un don réciproque et complémentaire, on voit quel bénéfice on peut 
tirer de cette vision dans la perspective d'une compréhension dynamique de notre présence au monde. Qu'est-ce que cela a à voir avec la poésie? Stanislas Breton l'énonce plus clairement que je ne saurais le faire: "Une poésie nous touche d'autant plus et nous apparaît d'autant plus réussie qu'elle réunit cette double qualité d'un exode qui nous dépayse et d'une profondeur d'enracinement qui nous invite à y demeurer." Tel serait le poète, immobile voyageur qui bâtit la demeure sans jamais cesser d'étreindre le corps du monde en son amoureux parcours. Et telle serait la poésie conciliatrice, comme un arbre majeur qui dans sa fixité même traverse le vent et qui dans l'infini de l'espace ainsi mesuré s'enrichit d'un chant. Tel serait le chant de l'homme, souffle du passage dans les frondaisons de sa présence.

Nous avons grand besoin aujourd'hui de l'âme, ce souffle agissant qui porte sur son aile le poids de la réalité et lève sur nos chemins quotidiens la poussière du sens. Anima ou spiritus, haleine vive, c'est de cet élan vers le mystère que tremble le poème. En 1946, Pier Jean Jouve écrivait ces mots: "Tout demande aujourd'hui au poète d'être le dépositaire spirituel. Tout lui demande de ramener la spiritualité dans un monde de matière bouchée. Tout lui demande de représenter la force spirituelle et de la communiquer dans un univers dont on peut tout dire y compris ceci, que la fin du monde approche. Le "petit véhicule" de la poésie est chargé des poids les plus lourds: il devient plus précieux à l'amour et à la justice à mesure que le char mécanique de l'iniquité mondiale grossit."

Ainsi va la poésie, médiatrice d'une spiritualité neuve qui chérit le corps douloureux du monde, ses gestes d'ombre et sa bouche de lumière. Pour ma part, j'ai espoir. Je sais des amis qui, sur le haut plateau cévenol, dans la vaste demeure de Cheyne, se font jour après jour les dépositaires humbles et obstinés d'une parole fervente, d'un chant nourri à l'épreuve quotidienne de l'arbre, de la pierre, de la neige et du silence, et qui de livres en livres construisent à leur place et sans faillir l'heure qui vient. Martine Mellinette et Jean-François Manier, éditeurs et créateurs, ont adressé à l'aube de l'année 1995 à ceux qui de près ou de loin accompagnent leur marche amoureuse ce message de Hölderlin qui fera la plus juste conclusion à cette quête du mystère où vous m'avez convié:

Les instants

où nous aurons enfin réussi à

nous parler vraiment,

les instants 
où le frère sera pour le frère

où l'homme sera pour l'homme

et l'âme humaine pour l'âme humaine

le témoignage évident d'une chose

sacrée et joyeuse,

ces instants-là seront dignes alors

de tous nos espoirs...

(Conférence, 27 février 1995) 